\title{
Deep Time-Delay Reservoir Computing: Dynamics and Memory Capacity
}

\author{
Mirko Goldmann, ${ }^{1,2,}$ a) ${ }^{2}$ Felix Köster, ${ }^{1}$ Kathy Lüdge, ${ }^{1}$ and Serhiy Yanchuk $^{3}$ \\ 1) Institute of Theoretical Physics, Technische Universität Berlin, D-10623, Germany \\ 2) Department of Mathematics, Humboldt-Universität zu Berlin, D-12489, Germany \\ ${ }^{3)}$ Institute of Mathematics, Technische Universität Berlin, D-10623, Germany
}

(Dated: 26 August 2020)

The Deep Time-Delay Reservoir Computing concept utilizes unidirectionally connected systems with timedelays for supervised learning. We present how the dynamical properties of a deep Ikeda-based reservoir are related to its memory capacity (MC) and how that can be used for optimization. In particular, we analyze bifurcations of the corresponding autonomous system and compute conditional Lyapunov exponents, which measure the generalized synchronization between the input and the layer dynamics. We show how the MC is related to the systems distance to bifurcations or magnitude of the conditional Lyapunov exponent. The interplay of different dynamical regimes leads to an adjustable distribution between linear and nonlinear MC. Furthermore, numerical simulations show resonances between clock cycle and delays of the layers in all degrees of the MC. Contrary to MC losses in single-layer reservoirs, these resonances can boost separate degrees of the $\mathrm{MC}$ and can be used, e.g. to design a system with maximum linear MC. Accordingly, we present two configurations that empower either high nonlinear MC or long time linear MC.

The brain-inspired reservoir computing paradigm manifests the natural computing abilities of dynamical systems. Inspired by randomly connected artificial neural networks called echo state networks, simple optic and opto-electronic hardware implementations were developed, opening the research for delay-based reservoirs. These systems show promising performance at different supervised machine learning tasks like timeseries forecasting, e.g., for the chaotic MackeyGlass attractor, but also in speech recognition. The implementations employ a dynamical node with delayed feedback, which can exhibit multidimensional complex dynamics. In delay-based reservoir computing, the nodes of the network are separated temporally, and the computation time correlates with the number of nodes. An ongoing search for systems with improved performance started, resulting in more complex implementations. In this paper, we analyse the concept of deep time-delay reservoir computing, where multiple delay systems, called layers, are coupled unidirectionally. Such a scheme enables a constant low computation time while the number of nodes increases via additional layers. We investigate the dynamics of the layers and explain the effects of their interplay, where the influence onto the computational capabilities are measured using the linear and nonlinear memory. By utilizing this interplay, we show a strong adaptability of the reservoirs performance and we show ways to optimize, e.g. the linear memory of a reservoir computer.

\footnotetext{
${ }^{a)}$ Electronic mail: mirko-goldmann@hotmail.de
}

\section{INTRODUCTION}

The introduction of the reservoir computing paradigm by Jaeger ${ }^{[1]}$ and Maass ${ }^{2]}$ independently gained considerable interest in supervised machine learning utilizing dynamical systems. The reservoir computing scheme contains three different parts: the input layer, the reservoir, and the output layer. The reservoir can be any dynamical system, like an artificial neural network but also a laser with self-feedback. The output layer is trained by a linear weighting of all accessible reservoir states, while the reservoir parameters are kept fixed. This simplification overcomes the main issues of the time expensive training of recurrent neural networks like exploding gradients and its high power consumption ${ }^{3}$.

Appeltant et al. $\frac{4}{}$ successfully implemented the RC scheme onto a single nonlinear node with a delayed selffeedback. In the input layer, time-multiplexing is used to create temporally separated virtual nodes. The reservoir dynamics are hereby given by a delay differential equation, which has been proven to exhibit rich highdimensional dynamics $5+8$. For the training, the temporally separated nodes are read out and weighted to solve a given task. The introduction of time-delay reservoir computing enabled simple optical and opto-electronic hardware implementations, which led to improvements of computation time scales for supervised learning 9110 . The delay-based reservoirs were successfully applied to a wide range of tasks, such as chaotic time series forecasting or speech recognition.

The success of single node delay-based reservoir computing has triggered interest into more complex network architectures, like coupled Stuart-Landau oscillators arranged in a ring topology 11 , single nonlinear nodes with multiple self-feedback loops of various length $\frac{12}{2}$, parallel usage of multiple nodes ${ }^{13}$ and multiple nonlinear nodes coupled in a chain topology 14 . Further, it was recently shown by Gallicchio et al! 15 , that echo state networks 
with multiple unidirectional coupled layers called deepESN provide a performance boost in comparison to their shallow counterpart. Various cascading reservoir setups

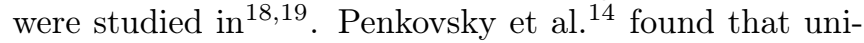
directional coupled delay systems are superior against bidirectional coupling for certain symmetric parameter choice.

In the following, we present a deep time-delay reservoir computing model, where we use asymmetrical layers and discuss their dynamical and computational properties. In contrast to the general deepESN scheme, the considered model possesses the same number of nodes in all layers, which is the result of our time multiplexing procedure for constructing a network from a time-delay system. Our setup is, in a certain sense, more simple than the cascading reservoirs considered in $\frac{18 \mid 19}{}$, since no output signals (e.g., linear regression) are generated at each layer separately. The input enters only the first layer, and each consecutive layer receives only the dynamical state of the previous layer. As a result, we do not perform sequential training of the layers.

The paper is structured as follows: In section III we present the system implementing deep time-delay reservoir computer and show its performance at predicting the chaotic Mackey-Glass attractor. Afterward in III] we study the dynamical properties of an autonomous twolayer system. The conditional Lyapunov exponent for a non-autonomous system is introduced in IV] The numerically calculated conditional Lyapunov exponent is then related to the linear and nonlinear memory capacity. In section VI the resonances of the clock cycle and the delaytimes are presented for two and three-layer systems.

\section{DEEP TIME-DELAY RESERVOIR COMPUTING}

\section{A. Model}

A deep time-delay reservoir computer (deepTRC) consists of $L \in \mathbb{N}$ nonlinear nodes with states $\boldsymbol{v}_{l}(t)$ for $l=$ $1,2, \ldots, L$. All $L$ nodes feature a self-feedback with a delay length $\tau_{l}>0$.

The nodes are coupled unidirectional, where the first node is the only which is feed by the task-specific input sequence $u(t)$. The coupling between the nodes is instantaneous, i.e., without delay. The nodes with their corresponding feedback loops are referred to as layers in the following because of the unidirectional topology and their high-dimensional dynamics. The dynamical evolution of a layer $l$ is given by:

$$
\dot{\boldsymbol{v}}_{l}(t)=F_{l}\left(\boldsymbol{v}_{l}(t), \boldsymbol{v}_{l}\left(t-\tau_{l}\right), J_{l}(t)\right)
$$

with $F_{l}(\ldots)$ being a nonlinear delay differential equation, and $J_{l}(t)$ the layer dependent input:

$$
J_{l}(t)=\left\{\begin{array}{l}
u(t) \text { for } l=1 \\
v_{l-1}^{1}(t) \text { else }
\end{array}\right.
$$

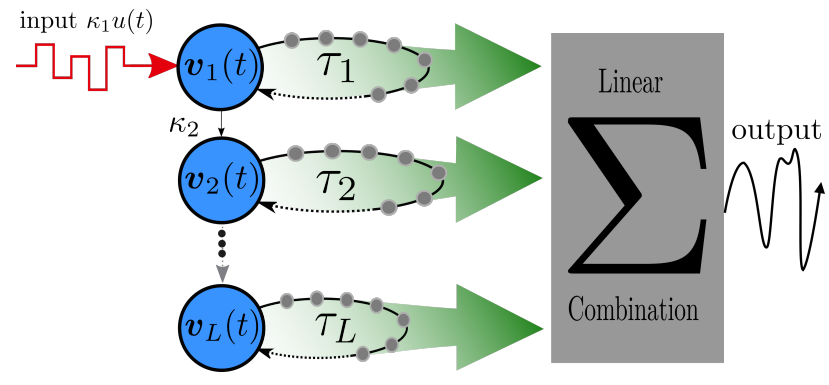

FIG. 1. Deep Time-Delay Reservoir Computer containing $L$ layers (blue) with delayed self-feedback, where the delay length $\tau_{l}$ can vary. The first layer $\boldsymbol{v}_{1}(t)$ is driven by the time multiplexed input sequence $\kappa_{1} u(t)$ whereas all other layers are driven by their previous layer weighted with $\kappa_{l}$. The output layer is given by linear weighting of all states.

In the following, we assume that the coupling to the layer $l$ is realized via the first component $x_{l-1}=v_{l-1}^{1}(t)$ of the dynamical variable $\boldsymbol{v}_{l-1}(t)$ of layer $l-1$. Timemultiplexing is used to transform the layers into high dimensional networks. The discrete input is given by the sequence $(s(k))_{k \in \mathbb{N}_{0}}, s(k) \in \mathbb{R}$. This sequence is transformed into the time-continuous input $u(t)$ as follows

$$
u(t)=u_{k, j}=s(k) m_{j} \text { for } t \in[k T+(j-1) \theta, k T+j \theta],
$$

where $T$ is the clock cycle and the scaling $m_{j}, j=$ $1, \ldots N_{V}=T / \theta$ determines a mask, which is applied periodically with the period $T$. Such a preprocessing method generates $N_{V}$ virtual nodes which are distributed temporally with a separation of $\theta=T / N_{V}$, see more details in 4111. The given deepTRC now contains $L$ layers with each having $N_{V}$ virtual nodes resulting in a total reservoir size of $N_{R}=L N_{V}$. The virtual nodes within the layers correspond to the values $x_{l}(k T+j \theta)=\boldsymbol{v}_{l}^{1}(k T+j \theta)$.

For the training of the deepTRC, all virtual nodes $x_{l}(k T+j \theta), j=1, \ldots, N_{V}$ of each layer $l=1, \ldots, L$ are read out. The virtual nodes are combined into the global state $X(k) \in \mathbb{R}^{N_{R}}$ given by

$$
X(k):=\left(\begin{array}{c}
X_{1} \\
X_{2} \\
\vdots \\
X_{N_{R}}
\end{array}\right):=\left(\begin{array}{c}
x_{1}(k T) \\
x_{1}(k T+\theta) \\
\vdots \\
x_{L}\left(k T+\left(N_{V}-2\right) \theta\right) \\
x_{L}\left(k T+\left(N_{V}-1\right) \theta\right)
\end{array}\right)
$$

In order to train for a given task $\hat{o}(k)$, the global state is weighted

$$
o(k)=W^{T} X(k)+c,
$$

where $c$ is a constant bias and the weights $W \in \mathbb{R}^{N_{R}}$ are determined via a linear regression with an optional Tikhonov regularization.

In the following, we will focus on the analysis of the recently introduced opto-electronic reservoir $[9|12| 14|20| 24$, 
which is governed by the equations:

$$
\begin{aligned}
\dot{x}_{l}(t) & =-x_{l}(t)-\delta_{l} y_{l}(t) \\
& +\beta_{l} \sin ^{2}\left(x_{l}\left(t-\tau_{l}\right)+\kappa_{l} J_{l}(t)+b_{l}\right), \\
\dot{y}_{l}(t) & =x_{l}(t),
\end{aligned}
$$

where $\kappa_{1}$ is the input gain and $\kappa_{l}, l>1$ are coupling strengths between the consecutive layers $l-1$ and $l$. Further, $\delta_{l}$ is a damping constant satisfying $\delta_{l} \leq \delta_{l+1}, \beta_{l}$ is the feedback gain and $b_{l}$ is a scalar phase shift of the nonlinearity. The dynamical variable of a layer becomes $\boldsymbol{v}_{l}=\left(x_{l}, y_{l}\right)^{T}$. According to the $\sin ^{2}$ nonlinearity, the system is referred as Ikeda time-delay system $14 \sqrt{25 \mid 26}$.

\section{B. Chaotic Time-Series Prediction}

In Fig. 2, we show the performance of the Ikeda based deepTRC at the prediction of the chaotic Mackey-Glass attractor, i.e., $\hat{o}(k)=s(k+\Delta n)$, where $s(t)$ is the timeseries of the Mackey-Glass system and $\Delta n$ determines how far into the future it shall be predicted. The chosen

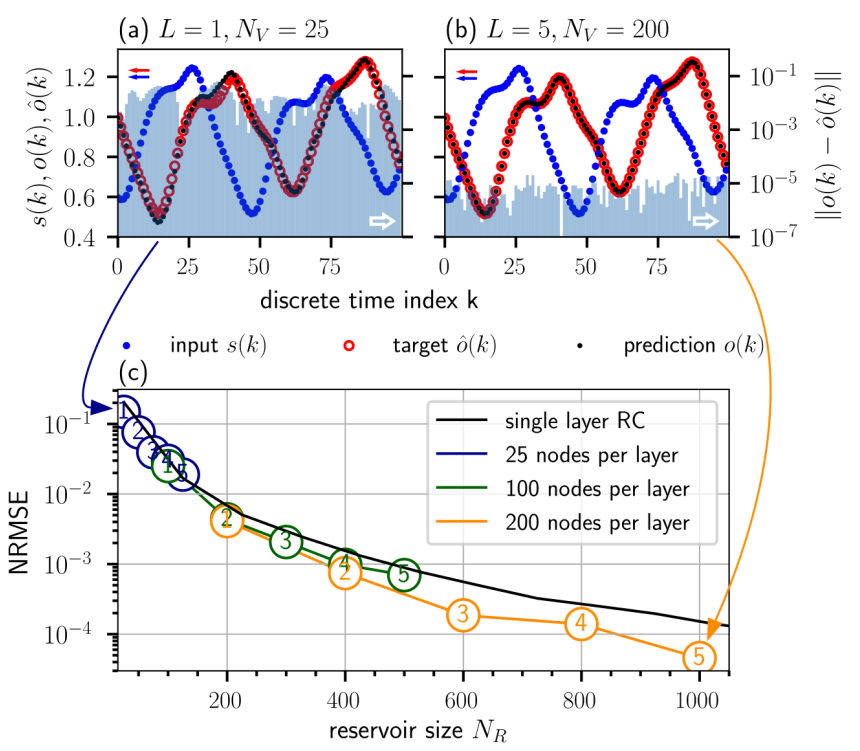

FIG. 2. Input $s(k)$, output $\hat{o}(k)$ and prediction $o(k)$ of the chaotic Mackey-Glass task of (a) a single-layer system with 25 virtual nodes $\left(N_{R}=25\right)$ and (b) a five-layer system with 200 virtual nodes per layer $\left(N_{R}=1000\right)$. Hereby the output $\hat{o}(k)$ is a temporal shift of the input sequence $\hat{o}(k)=s(k+34)$. (c) Performance comparison of deepTRCs with up to $L=5$ layers at the Mackey-Glass prediction task $\Delta n=34$ into future. The black line represents the performance of a single-layer TRC with the increase of virtual nodes $N_{V}$. The colored lines represent deepTRC, where the number in the circle marks the number of layers, e.g. orange circle with 3 means 3 layers with each having 200 nodes. For all shown simulations the separation of nodes was kept fixed at $\theta=1$. Accordingly the clock cycle $T=N_{V} \theta$ increases with the amount of virtual nodes $N_{V}$.

\begin{tabular}{|l|c|c|c|c|c|}
\hline & delay $\tau$ & damping $\delta$ & phase $b$ & feedback $\beta$ & coupling $\kappa$ \\
\hline \hline Layer 1 & 230 & 0 & 0.2 & 0.68 & 4.0 \\
\hline Layer 2 & 457 & 0.01 & 0.2 & 0.8 & 1 \\
\hline Layer 3 & 199 & 0.01 & 1.5 & 0.97 & 0.01 \\
\hline Layer 4 & 27 & 0.01 & 1.28 & 0.83 & 0.13 \\
\hline Layer 5 & 40 & 0.01 & 1.9 & 0.2 & 0.01 \\
\hline
\end{tabular}

TABLE I. Parameters of the five-layer deepTRC with the best performance $\left(\mathrm{NRMSE}=4.56 \times 10^{-5}\right)$ shown in Fig. 2 $(\mathrm{b})$. The deepTRC has $N_{V}=200$ virtual nodes per layer and the clock cycle is $T=200$.

prediction step $\Delta n=34$ corresponds to twice the delay time of the Mackey-Glass system which is shown in Fig. 2 (a) and (b). The parameters of the Mackey-Glass system are as in Jaeger et al.27. We simulated deepTRC with up to $L=5$ layers and varied the number of nodes per layer. Hereby, the separation of nodes is set to $\theta=1$ and will be kept fixed for all following simulations. Accordingly the clock cycle $T$ increases as the number of virtual nodes $N_{V}$ increases. A Bayesian optimization approach 28 was used to optimize the feedbackgains $\beta_{l}$, the delays $\tau_{l}$, coupling gains $\kappa_{l}$ and phase offsets $b_{l}$. Hereby, the Bayesian optimisation of deepTRC becomes much harder for deeper systems according to an increased amount of hyperparameters. In general, additional layers can improve the performance of deepTRC as compared to single-layer TRC as it is shown in (a) and (b). As a remark, the deepTRC enables a faster computation by a constant separation of nodes $\theta$, i.e a deepTRC with $L=5$ layers is 5 times faster than the single-layer TRC with the same amount of total nodes in line with a 5 times shorter clock cycle. For the evaluation of the performance an initial input of length $K_{\text {init }}=10^{4}$, a training length of $K_{\text {train }}=10^{4}$ and a testing length of $K_{\text {test }}=2000$ is used.

The parameters of the best deepTRC with $L=5$ layers are shown in table I. Note that the coupling gains of the last three layers $\kappa_{i}$ are small. This indicates that these layers might play the role of linear filtering of the input signal, with an additional mixing due to different delays. We discuss the role of the layers detailed in Sec. VIC.

\section{DYNAMICS OF AUTONOMOUS DEEPTRC}

The dynamics of a delay-based RC play an essential role for its performance $\frac{429}{4}$. In this section we consider an autonomous $L$-layer deepTRC by setting $J^{(1)}=0$.

The equilibrium of Eq. (6) are given as solutions of the following nonlinear system of equations

$$
\left\{\begin{array}{l}
x_{l}^{*}=\beta_{l} \sin ^{2}\left(x_{l}^{*}+b_{l}\right) \quad \text { if } \delta_{l}=0 \\
x_{l}^{*}=0, \\
y_{l}^{*}=\frac{\beta_{l}}{\delta_{l}} \sin ^{2}\left(\kappa_{l} x_{l-1}^{*}+b_{l}\right) \quad \text { if } \delta_{l}>0
\end{array}\right.
$$

Without further restriction we set $\delta_{1}=0$ and $\delta_{l>1}>$ 
0. System (6) can be linearised around this equilibrium, which leads to:

$$
\left(\begin{array}{c}
\dot{\boldsymbol{\xi}_{1}}(t) \\
\dot{\dot{\boldsymbol{\xi}}_{2}}(t) \\
\vdots \\
\dot{\boldsymbol{\xi}_{L}}(t)
\end{array}\right)=A\left(\begin{array}{c}
\boldsymbol{\xi}_{1}(t) \\
\boldsymbol{\xi}_{2}(t) \\
\vdots \\
\boldsymbol{\xi}_{L}(t)
\end{array}\right)+B\left(\begin{array}{c}
\boldsymbol{\xi}_{1}\left(t-\tau_{1}\right) \\
\boldsymbol{\xi}_{2}\left(t-\tau_{2}\right) \\
\vdots \\
\boldsymbol{\xi}_{L}\left(t-\tau_{L}\right)
\end{array}\right)
$$

where $\boldsymbol{\xi}_{l}(t)$ is the linearisation of $l^{\text {th }}$-layers dynamical variable $\boldsymbol{v}_{l}(t)$ and $\boldsymbol{\xi}_{l}\left(t-\tau_{l}\right)$ is the linearisation shifted by the delay of the layer.

The block matrix $A \in \mathbb{R}^{m \times m}, m=2 L$ is given by the sub-matrices $A_{l, l^{\prime}} \in \mathbb{R}^{2 \times 2}$

$$
A_{l, l^{\prime}}=\frac{d F_{l}\left(\boldsymbol{v}_{l}(t), \boldsymbol{v}_{l}\left(t-\tau_{l}\right), J_{l}(t)\right)}{d \boldsymbol{v}_{l^{\prime}}(t)},
$$

According to the unidirectional topology of the IkedadeepTRC matrix $A$ becomes a lower triangular block matrix. Further, the block matrix $B \in \mathbb{R}^{m \times m}$ can be calculated by:

$$
B_{l, l^{\prime}}=\frac{d F_{l}\left(\boldsymbol{v}_{l}, \boldsymbol{v}_{l}\left(t-\tau_{l}\right), J_{l}(t)\right.}{\left.d \boldsymbol{v}_{l^{\prime}}\left(t-\tau_{l^{\prime}}\right)\right)}
$$

Therefore, $B=\operatorname{diag}\left(B_{11}, B_{22}, \ldots, B_{L L}\right)$ becomes block diagonal. The sub-matrices of the autonomous Ikeda deepTRC (6) are given by:

$$
\begin{aligned}
A_{1,1} & =\left(\begin{array}{cc}
-1 & 0 \\
0 & 0
\end{array}\right), \quad A_{l, l}=\left(\begin{array}{cc}
-1 & -\delta_{l} \\
1 & 0
\end{array}\right) \text { for } l>1, \\
A_{l+1, l} & =\left(\begin{array}{cc}
\beta_{l+1} \kappa_{l+1} \nu_{l+1} & 0 \\
0 & 0
\end{array}\right), \quad B_{l, l}=\left(\begin{array}{cc}
\beta_{l} \nu_{l} & 0 \\
0 & 0
\end{array}\right)
\end{aligned}
$$

with $\nu_{1}=\sin \left(2\left(x_{1}^{*}+b\right)\right)$ and $\nu_{l>1}=\sin \left(2\left(\kappa_{l} x_{l-1}^{*}+b_{l}\right)\right.$. The Eigenvalues of the linearised system can therefore be calculated by solving the characteristic equation:

$$
0=|A-\lambda \mathbf{1}+\tilde{B}|
$$

with $\tilde{B}=\operatorname{diag}\left(B_{1,1} \exp \left(\lambda \tau_{1}\right), \ldots, B_{L, L} \exp \left(\lambda \tau_{L}\right)\right)$. This equation can further be simplified by using that the determinant of a lower triangular matrix is given by the product of the determinants of the block matrices:

$$
0=\prod_{l=1}^{L}\left|A_{l, l}-\lambda \mathbf{1}+B_{l, l} \exp \left(\lambda \tau_{1}\right)\right|
$$

The characteristic equation of our $L$-layer Ikeda deepTRC (6) is therefore given by:

$$
\begin{aligned}
0= & \left(-\lambda-1+\beta_{1} \nu_{1} \exp \left(\lambda \tau_{1}\right)\right) \\
& \left.\times \prod_{l=2}^{L}\left(\delta_{l}+\lambda\left(\lambda+1-\beta_{l} \nu_{l}\right) \exp \left(\lambda \tau_{l}\right)\right)\right)
\end{aligned}
$$

\begin{tabular}{|c|c|c|c|c|}
\hline Description & Parameter & Layer 1 & Layer 2 & Layer 3 \\
\hline \hline delay time & $\tau$ & 30 & 30 & 30 \\
\hline feedback gain & $\beta$ & 1.6 & 1.3 & 1.3 \\
\hline phase offset & $b$ & 0.2 & 0.2 & 0.2 \\
\hline coupling & $\kappa$ & 0.0 & 1 & 1 \\
\hline damping & $\delta$ & 0 & 0.01 & 0.01 \\
\hline initial value & $\boldsymbol{v}_{l, 0}$ & {$[0.07,0]^{T}$} & {$[0,0]^{T}$} & {$[0,0]^{T}$} \\
\hline
\end{tabular}

TABLE II. Parameter values used for the calculation of the bifurcation diagrams in Fig. 3. Additionally, the parameters are used for the conditional Lyapunov exponent in Fig. 4 and the memory capacity in Fig. 5 and 6, whereas there the input was enabled by setting the input gain $\kappa_{1}=0.01$.

For the details of the derivation we refer to Appendix B One can see that the eigenvalues of the linearised autonomous deepTRC are given by the combined set of the eigenvalues for the single layers.

In the following we study the dynamics of the two-layer deepRTC depending on the feedback gains $\beta_{1}$ and $\beta_{2}$. The remaining parameters are kept fixed to the values shown in Table 【.

In Fig. 3 (a) the equilibrium of the first layer $x_{1}^{*}$ is shown as a function of the feedback gain $\beta_{1}$. The layer exhibits two saddle-node bifurcations at $\beta_{1} \approx 1.15$ and $\beta_{1} \approx 1.3$ respectively; between these two points the first layer possesses two coexisting stable equilibria. Further, it reveals periodic solutions after the supercritical Hopf bifurcation at $\beta_{1} \approx 1.816$, and a period-doubling cascade resulting in chaotic dynamics at $\beta_{1} \approx 2.2$. The numerical part of the bifurcation diagram, i.e., the chaotic solutions of $x_{1}(t)$ in Fig. 3 (a) are computed using Heun's method. The stability and the location of the Hopf bifurcations are calculated using the derivation in Appendix B.

In Fig 3 (b) the bifurcation diagram of the second layer is shown for a constant feedback gain of the first layer $\beta_{1}=1.0$. The second layer reveals a subcritical Hopf at $\beta_{2} \approx 1.9$. The created unstable limit cycle becomes stable due to a saddle-node bifurcation at $\beta_{2} \approx 1.4$. Accordingly, a coexistence of a limit cycle and the stable equilibrium $x_{2}^{*}=0$ occurs in the range $\beta_{2} \in[1.4,1.9]$.

While the first layer can be considered separately, the second layer is driven by the first one. As a result, one has to study the whole system (B1) for analyzing the dynamics of the second layer. In Fig. 3 (c) the bifurcation diagram of the full two-layer system is obtained using the DDE-Biftool 30 . The bifurcations of the first layer occur as vertical lines (indicating saddle-node, Hopf and period-doubling bifurcations in blue, red and magenta) while the sub and supercritical Hopf and the fold bifurcations of the second layer occur as curved lines in the $\beta_{1}, \beta_{2}$ plane (green, red and orange).

In the following sections, the obtained bifurcation diagrams will be compared with the other characteristics of the reservoir that describe its memory capacity. In 

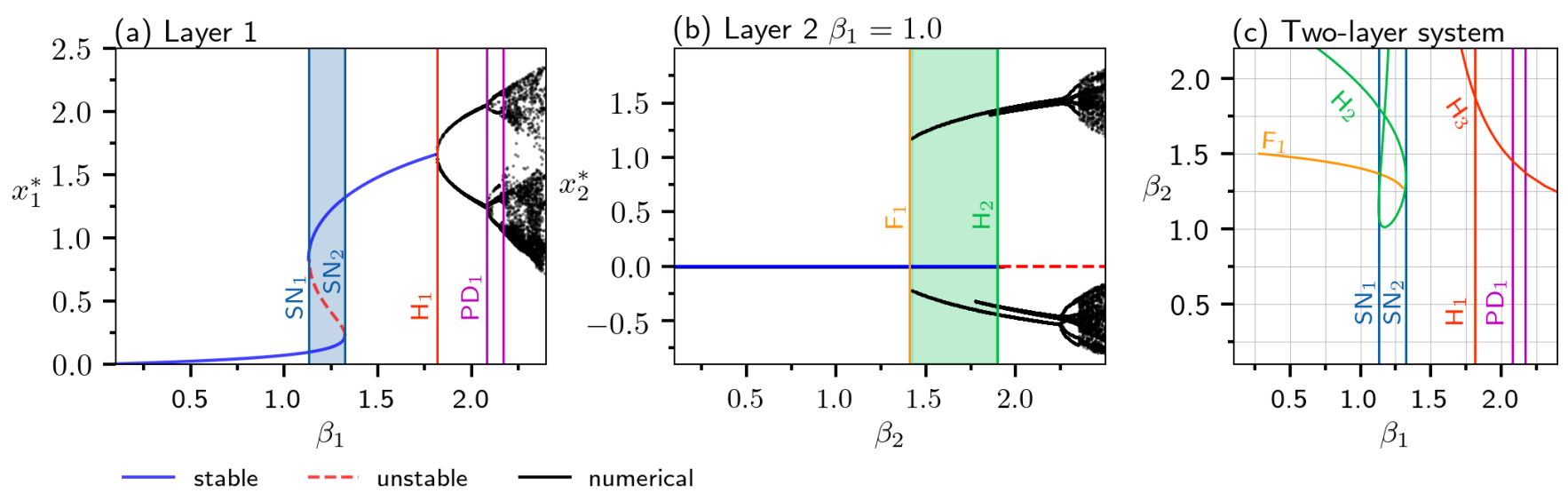

FIG. 3. (a) Bifurcation diagram of the first layer where the blue shaded area marks bi-stability, (b) bifurcation diagram of the second layer for constant $\beta_{1}=1.0$ where the green shaded area marks coexistence of a stable equilibrium $x_{2}^{*}=0$ and a stable limit cycle, (c) 2-parameter bifurcation diagram of the two-layer system. The stability and the Hopf bifurcations are computed using the analytical results presented in Appendix B all other bifurcations are computed using the DDE-Biftool. The used parameters are given in Table II Bifurcation legend: H (red) - supercritical Hopf, H (green) - subcritical Hopf, SN - saddle-node (blue),F - fold (orange) and PD - period doubling (magenta) bifurcation. For the periodic and chaotic parts of the bifurcation diagram in (a) and (b), the maxima and minima of the solutions are plotted.

particular, the next section investigates conditional Lyapunov exponents and shows how they restrict the parameter set where the RC can properly function.

\section{CONDITIONAL LYAPUNOV EXPONENT OF DEEP TIME DELAY RESERVOIRS}

To use the two-layer deep Ikeda reservoir we enable the input into the first layer by setting $J_{1}(t)=\kappa_{1} u(t)$ and solve the delay differential equation system (6) with $L$ initial history functions $\boldsymbol{v}_{l, 0}(s), s \in\left[-\tau_{l}, 0\right]$. The fading memory concept ${ }^{29}$ states that the reservoir needs to become independent of those history functions after a certain time. Therefore two identical reservoirs with different initial conditions need to approximate each other asymptotically. From a dynamical perspective, a reservoir has to show generalized synchronization to its input.

In the following, we check for generalized synchronization of two unidirectional coupled systems by estimating the maximal conditional Lyapunov exponent of the driven system. This is done by the auxiliary system method, where we initialize two identical systems with different initial conditions and drive both with the same input sequence. In order to provide comparability to later observations, the input sequence is drawn randomly from an uniform distribution $s(k) \sim \mathcal{U}[-1,1]$, and time multiplexing is used as described in equation (3) with $T=25$, $N_{V}=25$, and $\theta=1$. The conditional Lyapunov exponent then measures the convergence or divergence rate. If its maximal value is below zero, the state sequences will approximate each other asymptotically, and therefore the system shows generalized synchronization to the input system. If the exponent is positive, the systems diverge.

In order to calculate the conditional Lyapunov exponent we consider the distance between the solutions of two identical systems with different initial conditions: $\boldsymbol{v}(t)=\boldsymbol{v}\left(t, \phi_{\mathbf{0}}\right)$ and $\tilde{\boldsymbol{v}}(t)=\boldsymbol{v}\left(t, \boldsymbol{\phi}_{\mathbf{0}}^{\prime}\right)$ :

$$
\begin{aligned}
\dot{\boldsymbol{v}}(t) & =F\left(\boldsymbol{v}_{t}, u(t)\right) \\
\dot{\tilde{\boldsymbol{v}}}(t) & =F\left(\tilde{\boldsymbol{v}}_{t}, u(t)\right) \\
\dot{\boldsymbol{\mu}}(t) & =\dot{\tilde{\boldsymbol{v}}}(t)-\dot{\boldsymbol{v}}(t) \\
& =F\left(\boldsymbol{v}_{t}+\boldsymbol{\mu}_{t}, u(t)\right)-F\left(\boldsymbol{v}_{t}, u(t)\right)
\end{aligned}
$$

where

$$
\begin{aligned}
& F\left(\boldsymbol{v}_{t}, u(t)\right)= {\left[\begin{array}{c}
F_{1}\left(\boldsymbol{v}_{1}(t), \boldsymbol{v}_{1}\left(t-\tau_{1}\right), u(t)\right) \\
F_{2}\left(\boldsymbol{v}_{2}(t), \boldsymbol{v}_{2}\left(t-\tau_{2}\right), \boldsymbol{v}_{1}(t)\right) \\
\vdots \\
F_{L}\left(\boldsymbol{v}_{L}(t), \boldsymbol{v}_{L}\left(t-\tau_{L}\right), \boldsymbol{v}_{L-1}(t)\right)
\end{array}\right] } \\
& \boldsymbol{v}_{t}:=\boldsymbol{v}(t-s)=\left[\begin{array}{c}
\boldsymbol{v}_{1}(t-s) \\
\boldsymbol{v}_{2}(t-s) \\
\vdots \\
\boldsymbol{v}_{L}(t-s)
\end{array}\right], 0 \leq s \leq \tau_{\max }, \\
& \tau_{\max }=\max _{l=1 . . L} \tau_{l} \\
& \boldsymbol{\mu}_{t}=\boldsymbol{v}_{t}-\tilde{\boldsymbol{v}}_{t} .
\end{aligned}
$$

As a remark, $\boldsymbol{v}_{l}(t)$ gives the state of layer $l$ whereas $\boldsymbol{v}_{t}$ is a function of the $L$-layer system state defined over the interval given in (16). The evolution of the distance $\boldsymbol{\mu}(\boldsymbol{t})$ is now given by a set of delay differential equations. For small perturbations, we linearise equation (15):

$$
\dot{\boldsymbol{\mu}}(t)=A \boldsymbol{\mu}(t)+\beta B(t) \boldsymbol{\mu}_{t},
$$


where the linear part of $F$ was summarized into a constant matrix $A$, and the nonlinearity and the time varying input are included into $\beta B(t)$. According $\mathrm{tc}^{5}$ the solution of 17) can be estimated as

$$
\|\boldsymbol{\mu}(t)\| \leq e^{\lambda t} \text { const }
$$

where $\lambda$ is the conditional Lyapunov exponent of the nonautonomous reservoir.

For the numerical estimation of the conditional Lyapunov exponent, two equal non-autonomous systems with different initial conditions of the first layer $x_{1}(t), x_{1}^{\prime}(t)$ were evaluated. The input sequence was drawn from the uniform distribution $s(k) \sim \mathcal{U}[-1,1]$. The distances $\mu_{l}^{\text {num }}(t), l=1 . . L$, of the state sequences was calculated using the maximum norm over each delay interval $Q(q)=\left[(q-1) \tau_{l}, q \tau_{l}\right], q \in \mathbb{Z}$ :

$$
\mu_{l}^{\text {num }}\left(q \tau_{l}\right)=\sup _{t \in Q(q)}\left\|\boldsymbol{v}_{l}(t)-\boldsymbol{v}_{l}^{\prime}(t)\right\| .
$$

An exponential function was approximated accordingly to

$$
\mu_{l}^{\text {num }}\left(q \tau_{l}\right) \approx c e^{\lambda_{\max }^{(l)} q \tau_{l}},
$$

where $\lambda_{\max }^{(l)}$ determines the numerically approximated conditional Lyapunov exponent for layer $l$.

In Fig. 4 the numerical estimation of the conditional Lyapunov exponent is presented for a two-layer deepTRC. The parameters are as in Table II except the input gain, which was set to $\kappa_{1}=0.01{ }^{31}$ Here, the feedbackgains $\beta_{l}$ were scanned systematically for both layers.

In Fig 4 (a) the conditional Lyapunov exponent of the first layer is shown, which depends only on $\beta_{1}$. Here, the conditional Lyapunov exponent starts to increase with increasing $\beta_{1}$ and drops short behind the second saddle-node bifurcations $\mathrm{SN}_{2}$ at $\beta_{1}=1.32$ shown in Fig. 3 (a). In the region between the two saddle-node bifurcations $\mathrm{SN}_{1}$ and $\mathrm{SN}_{2}$ according to the bistability of the autonomous system two Lyapunov exponent were computed referring to different initial conditions. The dip at $\beta_{1} \approx 1.38$,close after the annihilation of the stable equilibrium $x_{1}^{*}=0.1$ with the unstable one, reveals the most negative Lyapunov exponent. After the Hopf bifurcation $\mathrm{H}_{1}$ in the first layer at $\beta_{1} \approx 1.8$ the conditional Lyapunov exponent becomes positive, and no generalized synchronization is possible. In other words, the system violates the fading memory condition for $\beta_{1}>1.8$.

The conditional Lyapunov exponent $\lambda_{\max }^{(2)}$ computed for the second layer reflects the dynamics of both layers. We observe from Fig. 4. that $\lambda_{\max }^{(2)}$ has, in general, a smaller magnitude than $\lambda_{\max }^{(1)}$. As a result, a perturbation of the first layer's initial condition stays longer in the system when a second layer is added. For larger values of $\beta_{1}$, the borders of the region, where the conditional Lyapunov exponent is negative, are determined by the Hopf bifurcations. The strong negative conditional Lyapunov exponent in the range $\beta_{1} \in[0.1,1], \beta_{2} \in[0.5,0.75]$ (a)

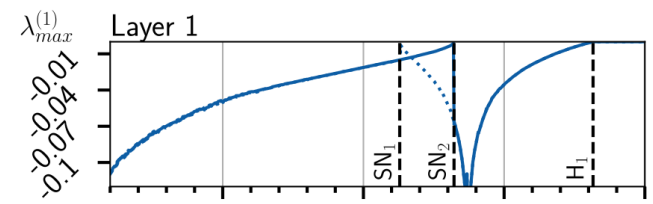

(b)

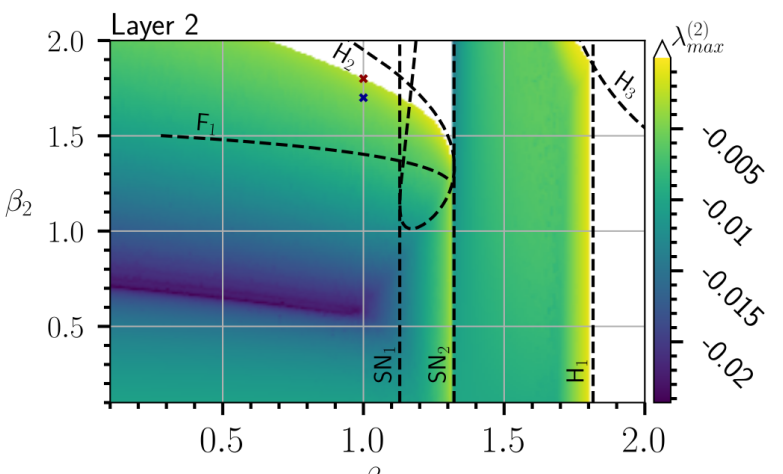

(c)

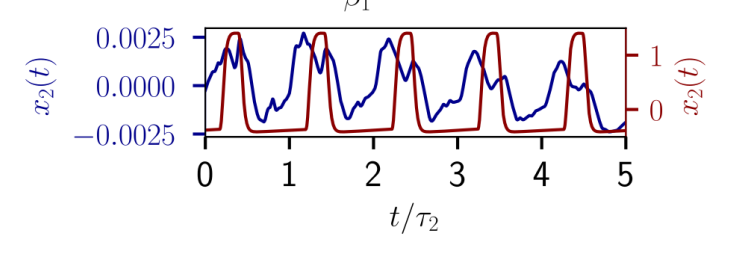

FIG. 4. Numerical estimations of conditional Lyapunov exponents of (a) $\lambda_{\max }^{(1)}$ for the first layer as a function of the feedback gain $\beta_{1}$, and (b) $\lambda_{\max }^{(2)}$ for the second layer color coded in the parameter plane $\left(\beta_{1}, \beta_{2}\right)$. Black dashed lines show the positions of the bifurcations shown in Fig. 3 In panel (a), two lines (solid and dotted) correspond to two different conditional Lyapunov exponents, which are obtained for two different sets of initial conditions of the first layer. They appear due to bistability of the layer dynamics for $\beta_{1}$ between the saddle-node bifurcations. In (b) the initial conditions are set equally to the calculated conditional Lyapunov exponent corresponding to the solid line in (a). In (c) the transients of the second layer $x_{2}(t)$ are shown for feedback gains $\beta_{1}=1, \beta_{2}=1.7$ (blue line) and $\beta_{1}=1, \beta_{2}=1.8$ (red line) which further correspond to the colored crosses in (b). The latter reveals a periodic oscillation of the length of the delay $\tau_{2}$, leading to loss of generalized synchronization shown in (b).

is due to the so-called exceptional point ${ }^{32}$, were two negative real eigenvalues coalescence. In the parameter range $\beta_{1} \in[0.7,1.3], \beta_{2} \in[1.5,2]$ the second layer losses generalized synchronization before reaching the subcritical Hopf bifurcation. We assume that due to the ongoing drive of the system, the second layer is pushed into the basin of attracting of the coexisting limit cycle, shown in Fig. 4(c) (red line). Such periodic oscillations lead to a loss of generalized synchronization before reaching the subcritical Hopf $\mathrm{H}_{2}$. Note that the observed oscillations are strongly nonlinear and their shape has a "switching" property known for such type of systems ${ }^{33}$. 


\begin{tabular}{|c|c|c|}
\hline Description & Parameter & Value \\
\hline \hline separation of nodes & $\theta$ & 1 \\
\hline clock cycle & $T$ & 25 \\
\hline virtual nodes per layer & $N_{V}$ & 25 \\
\hline initial steps & $K_{\text {init }}$ & $10^{5}$ \\
\hline training steps & $K_{\text {train }}$ & $10^{5}$ \\
\hline MC threshold $\left(N_{R}=50\right)$ & $C_{t h}$ & 0.012 \\
\hline MC threshold $\left(N_{R}=75\right)$ & $C_{t h}$ & 0.018 \\
\hline
\end{tabular}

TABLE III. Parameter values used for computation of the memory capacity in Fig. 5 and Fig. 6 .

\section{CONDITIONAL LYAPUNOV EXPONENTS VERSUS MEMORY CAPACITY}

In this section, we systematically investigate the relation between the conditional Lyapunov exponent and the MC for two-layer deepTRC. The MC measures how the reservoir memorizes and transforms previous input ${ }^{29134 \mid 35}$, for more details about MC and a definition, we refer to Appendix C.

In Fig. 5. we show the linear, quadratic, cubic, and total MC for the parameter values as in Table III The maximal total memory capacity $M C_{\text {tot }}=50$ can be reached in a wide parameter range, as shown in Fig. 5 (d), except for the regions with periodic solutions or strongly negative conditional Lyapunov exponent. In all four panels, a clear drop of the MC is visible close after the supercritical Hopf bifurcation in the first layer due to the violation of fading memory, i.e., in layer 1 at $\beta_{1} \approx 1.816$. Also the drop of MC occurs before reaching the subcritical Hopf in layer two, which is in agreement with the loss of generalized synchronization shown in Fig. 4. More specifically, we observe the following features:

- A large memory capacity, which is necessary for a reservoir computer to perform its tasks, is observed in the regions where conditional Lyapunov exponent is negative, and the fading memory condition is satisfied.

- The highest linear MC can be achieved close to the bifurcations where the conditional Lyapunov exponent is negative and small in absolute value. In such a case, the linear information of the input stays longer in the system.

- With the decreasing of the conditional Lyapunov exponent, the linear $\mathrm{MC}$ is decreasing, and $\mathrm{MC}_{2}$ starts dominating. With the further decrease of conditional Lyapunov exponent, the third-order $\mathrm{MC}$ becomes dominant. We remark that there is always a trade-off between the MC of different degrees since the total MC bounds their sum.

Concluding, different dynamical regimes of a deepTRC can boost different degrees of the MC.

\section{RESONANCES BETWEEN DELAYS AND CLOCK CYCLE}

As recently shown analytically and numerically by Stelzer et al $\stackrel{36}{ }$, resonances between delay-time $\tau$ and clock cycle $T$ lead to a degradation of linear memory capacity due to parallel alignment of eigenvectors of the underlying network. This effect was later shown in all degrees of the memory capacity by Harkhoe et al ${ }^{35}$ and Köster et al ${ }^{34}$ independently. This loss of total memory capacity at all resonances $a \tau \approx b T, a, b \in \mathbb{N}$ of delaytime and clock cycle further results in less performance at certain tasks.

In the following, we analyze this effect for two and three layer deepTRC via computing the single degrees of memory capacity up to the cubic degree while scanning the delays $\tau_{1}, \tau_{2}$, and $\tau_{2}, \tau_{3}$, respectively. The system parameters are as in Table II and the simulation parameter are shown in Table III.

\section{A. Two-Layer deepTRC}

In Figs. 6 (a)-(d) the numerically computed memory capacities are shown as a function of the delays of a twolayer deepTRC. Resonances between the delays $\tau_{1}, \tau_{2}$, and the clock cycle $T=25$ are present in all shown degrees of the MC. Further, resonances of the two delays occur as diagonals in the plot, with the main diagonal being dominant. In contrast to the off-diagonal resonances, the $\tau_{1}=\tau_{2}$ resonance broadens with higher delays. The total memory capacity exhibits weak degradations at the diagonal delay-delay and the clock cycle-delay resonances. The comparison between the linear and nonlinear MC reveals the trade-off between both, where the linear MC becomes dominant at $\tau_{1} \gtrsim 75$ and $\tau_{2}<\tau_{1}$.

In contrast to the reported linear MC degradations of the delay--clock cycle resonances for single-layer TRC, a new effect is visible in Fig. 6 (a), where the $\tau_{2}=k T$ resonance crosses the main diagonal. We observe that for fixed $\tau_{2}=k T$, when $\tau_{1}$ increases, the linear $\mathrm{MC}$ is degraded for $\tau_{1}<\tau_{2}$, while it is boosted for $\tau_{1}>\tau_{2}$.

In the following, we present an explanation of a boosted linear MC of a two-layer deepTRC for $\tau_{1}>$ $\tau_{2}=2 T$. For this, we compute the linear recallability 27 $M C(n) \in[0,1]$ of inputs $n$-steps into the past and separate between the two layers. Hereby, $M C(n)=1$ means the reservoir can fully recover the input from $n$ clock cycles ago. In Fig. 7 (a) the recallability of both layers is presented in different colour. As shown here, increasing $\tau_{1}$ for a single layer TRC leads to an improved recallability from inputs farther in the past. For comparison, at $\tau_{1}>50$, the single-layer TRC is able to recall inputs up to $n \approx 15$. For $\tau_{1}>2 T$, the linear MC splits up into small intervals with a high MC alternated with intervals of almost no MC, visible as blue rays in Fig. 7 . The length of the intervals of no MC further increases for longer delay $\tau_{1}$, where the frequency can be estimated as 

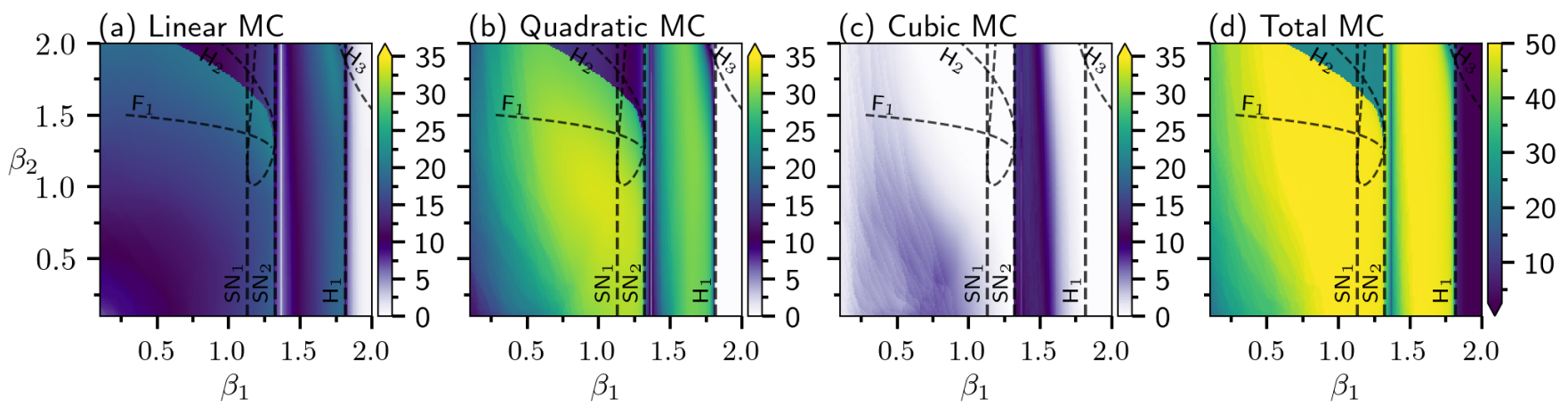

FIG. 5. Dependence of the memory capacity (MC) on the feedback gains parameters $\beta_{1}$ and $\beta_{2}$. In (a)-(c) the linear, quadratic and cubic MC is color coded while (d) shows the total MC. The black dashed lines show the positions of the bifurcations shown in Fig. 3. The parameters are as given in Table II.
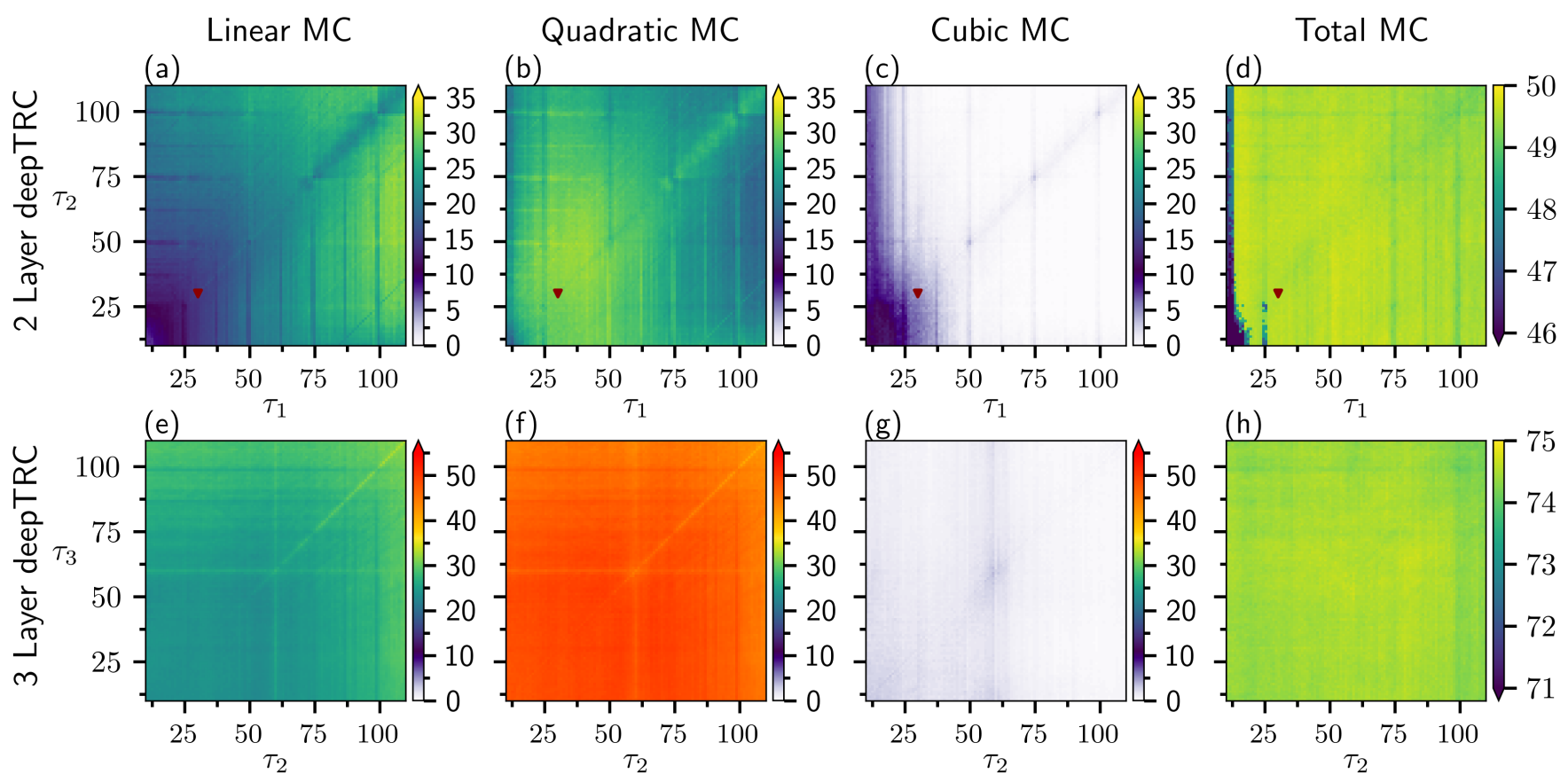

FIG. 6. (a) -- (c) Memory capacities (MC) of the linear, quadratic, cubic degree and (d) total MC plotted color coded for the two-layer deepTRC. The reservoir size is $N_{R}=50$ and the clock cycle is $T=25$. The red triangles mark the delay setting used in Fig. 5 (e) -- (g) show the linear, quadratic, cubic degree of the MC and (h) the total MC color coded for the three-layer deepTRC with $\tau_{1}=60$ fixed. The reservoir size is $N_{R}=75$ and the clock cycle $T=25$. The feedback gains $\beta_{l}$ are given in table III The resolution of the delay scan is $\Delta \tau_{i}=0.5$.

$\tau_{1} / T$

The addition of a second layer with a delay of $\tau_{2}=2 T$, while the delay of the first layer is $\tau_{1}<2 T$, increases the length of the recallability up to $n=20$. Furthermore, for $\tau_{1}>2 T$ the intervals of low MC are augmented by the $\mathrm{MC}$ of the second layer. Here, the areas with a high recallability in layer 1 (blue) are augmented by areas of high recallability in layer 2 (red), which results in a overall higher linear MC. Accordingly we call the appearing phenomenon augmentation effect. Again, resonance effects at $k \tau_{1}=m T$ occur, resulting in degradations.

\section{B. Three-layer deepTRC}

In Figs. 6 (e)-(h), we show the MC of a three-layer deepTRC with a reservoir size of $N_{R}=75$. The delay of the first layer is fixed at $\tau_{1}=60$, and the delay plane $\left(\tau_{2}, \tau_{3}\right)$ is scanned. The linear and quadratic MC exhibit strong resonances at $\tau_{2}=\tau_{1}=60, \tau_{3}=\tau_{1}=60$, which increase the linear MC. The resonances with multiples of the clock cycle occur as well, but they are less prominent with $\tau_{2}, \tau_{3}<\tau_{1}$. The resonances between $\tau_{2}$ and $\tau_{3}$ occur as the main diagonal in the plots. In comparison to the 
two-layer case, here the memory is more regular in the shown range $\tau_{2,3} \in[10,120]$. Changes in the memory are more strongly influenced by the first layer delay $\tau_{1}$. The cubic MC becomes maximal with the delays of all three layers being equal. The total $\mathrm{MC}$ is almost equal for all $\tau_{2}$ and $\tau_{3}$ showing a small general loss and with weak degradations at resonances with the clock cycle for $\tau_{2}, \tau_{3}>\tau_{1}$.

\section{Memory Capacity Distribution of deepTRC}

In this section, we show the role of multiple layers of deepTRC and we show ways how to systematically create certain memory in the a deepTRC. In particular, we present two configurations: the first one allows using the deep layers for producing large higher-order MC; the second configuration produce an increasing in the linear MC with the growing number of layers.

We start with a single-layer TRC. Fig. 8 (a) shows the MC distribution with an increasing of the number of virtual nodes $N_{V}$. The increase of the nodes number leads mainly to an increase of quadratic $\mathrm{MC}$, and, starting from $N_{V}=100$, a slow increase of the cubic MC. As a remark, increasing the virtual nodes of a single-layer TRC changes the clock cycle and, therefore, we adjusted the delay to $\tau_{1}=1.2 T$.

In Fig. 8 (b), we keep $N_{V}=25$ fixed and add layers with the same number of nodes to the deepTRC. The delay of all layers is kept fixed at $\tau_{l}=27$ and the clock cycle is $T=25$. We observe that additional layers lead to an increasing of nonlinear MC of higher orders.

The third configuration is similar to (b) but now the delays were varied in order to boost only the linear MC. Here, the augmentation effect shown in Fig. 7 was extended to $L>2$ layers by using the phenomenologically

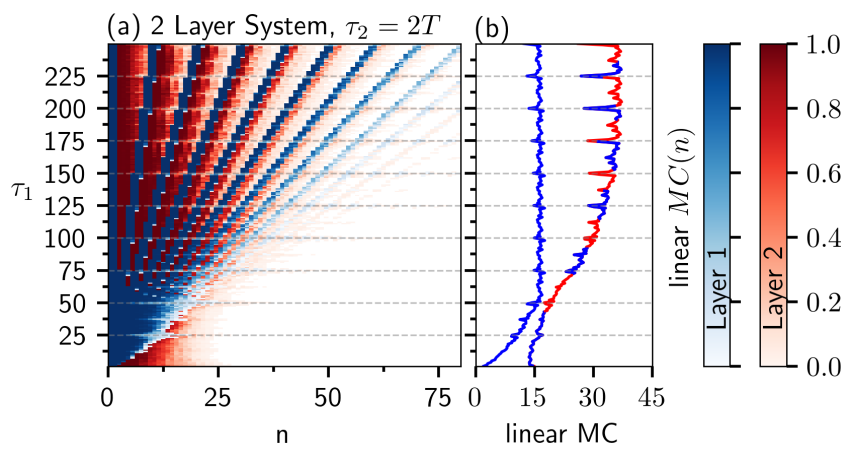

FIG. 7. (a) Linear recallability $\mathrm{MC}(\mathrm{n})$ as a function of the recalled past input $n$ and the delay $\tau_{1}$ depicted as color codes for the single layer system (blue) and an additional second layer (red). Here, the second layer augments the intervals with low $\mathrm{MC}$ of the first layer. (b) Overall linear memory capacity of the single-layer system (blue) and the strongly superior two-layer system (red-blue) for increasing delay $\tau_{1}$. The latter benefits from the appearing augmentation effect.

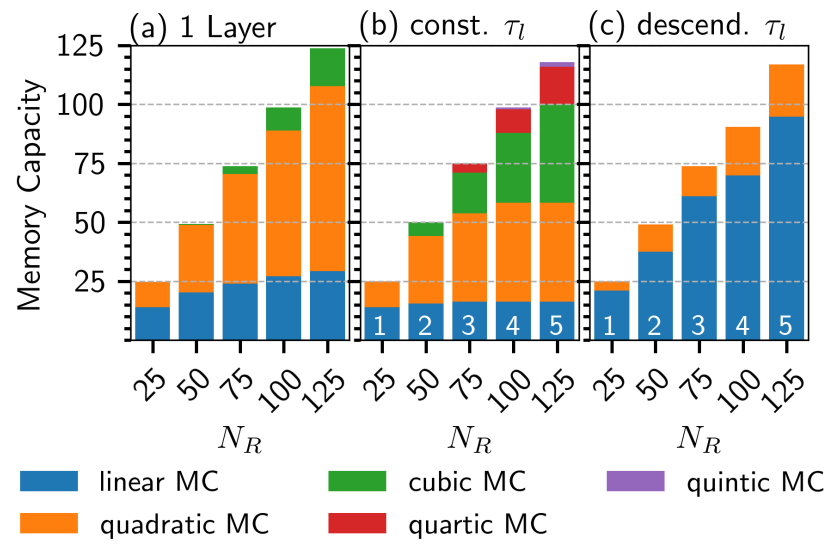

FIG. 8. Distribution of the memory capacities (MC) of different degrees (different colors within the bars) for (a) a singlelayer TRC, (b) deepTRC with constant delays $\tau_{l}=27$ and (c) deepTRC with optimal linear MC using descending delays $\tau_{l}=2^{L-l} \cdot 60$, e.g. the five layer deepTRC in (c) has the following delays: $\tau_{1}=960, \tau_{2}=480, \tau_{3}=240, \tau_{4}=120$ and $\tau_{5}=60$. The white number at the foot of the bar marks the number of layers $L$.

found rule $\tau_{l}=2^{(L-l)} \cdot 60$. The last layer has a delay $\tau_{1}=60$ because here a single layer shows its largest linear MC before splitting into rays. As shown in Fig. 8(c), this method produces large linear MC, while suppressing higher order MC.

Possible combinations of the presented delay configurations can be a good method for adjusting a deepTRCs memory to specific tasks. The deepTRC configurations in Fig. 8 show small general losses of total MC with a increasing number of layers, which is caused by linear dependence of different nodes.

\section{CONCLUSION}

We analyzed a Deep Time-Delay Ikeda System and presented a relation between the dynamics of the autonomous deepTRC and the numerically computed conditional Lyapunov exponent. We showed a correlation between MC and conditional Lyapunov exponents. A high linear MC is observed for small negative conditional Lyapunov exponent. With decreasing of the Lyapunov exponent, different degrees of $\mathrm{MC}$ are sequentially activated. Further, we investigated the clock cycle-delay resonances in different layers as well as delay-delay resonances. The degradation of the total MC at clock cycle delay resonances was numerically shown. We explained the boost of the linear MC at specific delay-clock cycle resonances by an augmentation effect. Additionally, we used the gained information to present two general delay configurations, one with an increasing nonlinear $\mathrm{MC}$ and one with boosted linear MC. These configurations provide a variability superior to a single-layer TRC. They provide a potential for building general deepTRC, 
which are oriented at different tasks due to a broad MC spectrum. We could verify that the deepTRC concept is a promising architecture for fast and variable reservoir computing.

\section{ACKNOWLEDGEMENTS}

The authors would like to thank Florian Stelzer for fruitfull discussions. M.G acknowledges financial support provided by the Deutsche Forschungsgemeinsschaft (DFG, German Research Foundation) through the IRTG 1740. K.L. and F.K. acknowledge support from the Deutsche Forschungsgemeinschaft in the framework of the CRC910. S.Y. acknowledges the financial support by the Deutsche Forschungsgemeinschaft - Project 411803875 .

\section{DATA AVAILABILITY}

The data that support the findings of this study are available from the corresponding author upon reasonable request.

\section{Appendix A: Normalised Root Mean Squared Error}

The performance at a certain task is measured using the normalized root mean squared error (NRMSE) given by:

$$
N R M S E=\sqrt{\frac{1}{K_{\mathrm{tst}}} \sum_{k=0}^{K_{\mathrm{tst}}} \frac{\|o(k)-\hat{o}(k)\|}{\operatorname{var}(o(k))}}
$$

where $K_{\text {tst }}$ is the length of the testing sequence, $o(k)$ is the reservoir output and $\hat{o}(k)$ are the task-dependent desired outputs. Further, $\operatorname{var}(\hat{o}(k))$ is the variance of the desired output and $\|\cdot\|$ is the Euclidean norm.

\section{Appendix B: Stability and Hopf Bifurcation Analysis}

This section gives a detailed analysis of the autonomous dynamics of the two-layer deep Ikeda timedelay system given in (6) which we repeat here for convenience:

$$
\begin{aligned}
\dot{x}_{1}(t)= & -x_{1}(t)+\beta_{1} \sin ^{2}\left(x_{1}\left(t-\tau_{1}\right)+b_{1}\right), \\
\dot{x}_{2}(t)= & -x_{2}(t)-\delta_{2} y_{2}(t) \\
& +\beta_{2} \sin ^{2}\left(x_{2}\left(t-\tau_{2}\right)+\kappa_{2} x_{1}(t)+b_{2}\right), \\
\dot{y}_{2}(t)= & x_{2}(t) .
\end{aligned}
$$

By setting the derivates to zero, the equations for the equilibrium are given by:

$$
\begin{aligned}
& x_{1}^{*}=\beta_{1} \sin ^{2}\left(x_{1}^{*}+b_{1}\right), \\
& x_{2}^{*}=0, \\
& y_{2}^{*}=\frac{\beta_{2}}{\delta_{2}} \sin ^{2}\left(\kappa_{2} x_{1}^{*}+b_{2}\right),
\end{aligned}
$$

where the equilibrium $x_{1}^{*}$ of the first layer cannot be given explicitly. In order to check the stability we linearise the system at its equilibrium leading to:

$$
\begin{aligned}
\dot{\xi}_{1}= & -\xi_{1}(t)+\beta_{1} \sin \left(2\left(x_{1}^{*}+b_{1}\right)\right) \xi\left(t-\tau_{1}\right) \\
\dot{\xi}_{2}= & -\xi_{2}+\beta_{2} \kappa_{2} \sin \left(2\left(\kappa_{2} x_{1}^{*}+b_{2}\right)\right) \xi_{1}(t) \\
& +\beta_{2} \sin \left(2\left(\kappa_{2} x_{1}^{*}+b_{2}\right)\right) \xi_{2}\left(t-\tau_{2}\right)-\delta \eta_{2}(t) \\
\dot{\eta}_{2}= & \xi_{2}(t)
\end{aligned}
$$

This can be rewritten into a lower triangular block matrix form as shown in section III and therefore the resulting characteristic equation is given by:

$$
0=\underbrace{\left(-\lambda-1+\beta_{1} \nu_{1} e^{-\lambda \tau_{1}}\right)}_{\text {Layer } 1} \cdot \underbrace{\left.\left(\delta+\lambda\left(\lambda+1-\beta_{2} \nu_{2}\right) e^{-\lambda \tau_{2}}\right)\right)}_{\text {Layer } 2}
$$

with $\nu_{1}=\sin \left(2\left(x_{1}+b\right)\right)$ and $\nu_{2}=\sin \left(2\left(\kappa x_{1}+b_{1}\right)\right.$ and lambda being the eigenvalues of the autonomous deepTRC.

An equilibrium $\left(x_{1}^{*}, x_{2}^{*}, y_{2}^{*}\right)$ is asymptotically stable if the real part of every solution of the characteristic equation is negative:

$$
\operatorname{Re}\left(\lambda_{i}\right)<0 \forall i
$$

what we check for in the following for layer 1 and layer 2 separately.

\section{Stability of Layer 1}

The eigenvalues of the first layer are roots of the equation $-\lambda-1+\beta_{1} \nu_{1} e^{-\lambda \tau_{1}}=0$, and they are given by the Lambert- $W$ function:

$$
\lambda_{i}=\frac{1}{\tau_{1}} W_{i}\left(\beta_{1} \nu_{1} \tau_{1} e^{\tau_{1}}\right)-1
$$

with $W_{i}(\ldots)$ being the $i$-th order of the Lambert- $W$ function.

The stability of the equilibrium point will change if the eigenvalues cross the imaginary axis. Therefore we set $\lambda=i \omega$

$$
i \omega=-1+\beta_{1} \nu_{1} e^{i \phi}
$$


where $\phi=\omega \tau_{1}$. Separating the real and imaginary part we can rewrite this into:

$$
\begin{aligned}
& 1=\beta_{1} \nu_{1} \cos (\phi), \\
& \omega=\beta_{1} \nu_{1} \sin (\phi),
\end{aligned}
$$

using the absolute values this leads to:

$$
\begin{array}{r}
\omega_{H} \tau_{1}^{*}=\phi^{*}=\arccos \left(\frac{1}{\beta_{1} \nu_{1}}\right), \\
\omega_{H}=\sqrt{\beta_{1}^{2} \nu_{1}^{2}-1}, \\
\tau_{1}^{*}=\frac{\arccos \left(\frac{1}{\beta_{1} \nu_{1}}\right)}{\sqrt{\beta_{1}^{2} \nu_{1}^{2}-1}},
\end{array}
$$

with $\tau_{1} \leq \tau_{1}^{*}$ being stable and $\tau_{1}>\tau_{1}^{*}$ being unstable due to a Andronov-Hopf bifurcation.

\section{Stability of Layer 2}

For the second layer we analyse the second term of the characteristic equation given by:

$$
\delta+\lambda\left(\lambda+1-\beta_{2} \nu_{2}\right) e^{\lambda \tau_{2}}=0 .
$$

By substituting $\lambda=i \omega$, it is straightforward to find the frequencies, at which the equation can cross the imaginary axis

$$
\bar{\omega}^{2}=-\frac{1-2 \delta-\left(\beta_{2} \nu_{2}\right)^{2}}{2} \pm \frac{\sqrt{\left(1-2 \delta-\left(\beta_{2} \nu_{2}\right)^{2}\right)^{2}-\delta^{2}}}{4} .
$$

The Hopf Bifurcation of the second layer occur in regions where: $\bar{\omega}_{ \pm}>0$. Denoting:

$$
\begin{array}{r}
\phi(\omega)=\arctan 2\left(\operatorname{sign}\left(\beta_{2} \nu_{2}\right) \omega, \delta-\omega^{2}\right), \\
\phi\left(\omega_{-}\right)=\omega_{-} \tau_{H}+2 \pi k, k \in \mathbb{Z} .
\end{array}
$$

the delay values for stabilizing $\tau_{H, k}-$ and destabilizing $\tau_{H, k}+$ Hopf bifurcations are given as:

$$
\begin{aligned}
\tau_{H, k}- & =-\frac{\phi\left(\omega_{-}\right)}{\omega_{-}}+\frac{2 \pi k}{\omega_{-}}, \\
\tau_{H, k}+ & =-\frac{\phi\left(\omega_{+}\right)}{\omega_{+}}+\frac{2 \pi k}{\omega_{+}} .
\end{aligned}
$$

\section{Appendix C: Memory Capacity}

The task-independent memory capacity (MC) introduced by Dambré et al! ${ }^{29}$ determines how a dynamical system memorizes previous inputs and how it further transforms them. The total $\mathrm{MC}$ is given by the sum over all degrees $d=1, \ldots, \infty, d \in \mathbb{N}$ of $\mathrm{MC}$.

$$
M C_{\text {tot }}=\sum_{d=1}^{D=\infty} M C_{d}
$$

In the following $d=1$ refers to the linear and $d>1$ to the nonlinear MC (quadratic, cubic, ... ). Hereby, the linear $\mathrm{MC}$ is given by a simple linear recall of past inputs, whereas the nonlinear gives evidence about which computation of past inputs are performed by the system. Further, it was proven that the read-out dimension of a system bounds the maximal reachable total $\mathrm{MC} M C_{\text {tot }} \leq$ $N_{R}$. According to this fact, a trade-off between the linear and nonlinear MC can be obtained.

The MC can be calculated via the correlation between input and the reservoir states:

$$
\begin{aligned}
C[x, \hat{o}] & =\frac{\langle\hat{o} X\rangle_{T}\left\langle X^{T} X\right\rangle_{K}^{-1}\left\langle X^{T} \hat{o}\right\rangle_{K}}{\left\langle\hat{o}^{2}\right\rangle_{K}}, \\
\langle\hat{o}\rangle_{K} & =\frac{1}{K} \sum_{t=1}^{K} \hat{o}(t)
\end{aligned}
$$

where $\langle\ldots\rangle_{K}$ is the average value over the time $K,,^{-1}$ is the inverse and ${ }^{T}$ the transpose of the matrix. The calculation of the MCs via the correlation given in (C3) is biased due to statistics and this bias strongly depends on the length of $K$. Therefore we manually set a threshold meaning that no MCs below this threshold are regarded. As suggested by Dambre et al 29 , the input values $s(k)$ were drawn from a uniform distribution $s \sim \mathcal{U}[-1,1]$. In order to compute the different degrees of $\mathrm{MC}$ we used the set of Legendre polynomials $\mathcal{L}_{p}$, which provide orthogonality over the given input range.

For example, a target of the cubic degree $d=3$ and three variables is given by:

$$
\begin{aligned}
\hat{o}(k)= & \mathcal{L}_{1}\left(k-n_{1}\right) \mathcal{L}_{1}\left(k-n_{2}\right) \mathcal{L}_{1}\left(k-n_{3}\right), \\
& n_{1}<n_{2}<n_{3} .
\end{aligned}
$$

In order to find all appearing $C_{d}(\ldots)$ a maximal step into the past of $n_{\max }=1000$ was set.

${ }^{1}$ H. Jaeger, GMD Rep. pp. 1-47 (2001), ISSN 18735223. I

${ }^{2}$ W. Maass, T. Natschläger, and H. Markram, Neural Comput. 14, 2531 (2002), ISSN 0899-7667, URL http://www.ncbi.nlm. nih.gov/pubmed/12433288 I]

${ }^{3}$ P.J. Werbos and P. J. Werbos, Proc. IEEE 78, 1550 (1990), URL http://ieeexplore.ieee.org/document/58337/?reload=true I

${ }^{4}$ L. Appeltant, M. C. Soriano, G. Van Der Sande, J. Danckaert, S. Massar, J. Dambre, B. Schrauwen, C. R. Mirasso, and I. Fischer, Nat. Commun. 2, 466 (2011), ISSN 20411723, URL http://dx.doi.org/10.1038/ncomms1476 I] II A III

${ }^{5} \mathrm{~J} . \quad$ K. Hale and S. M. V. Lunel, Introduction to Functional Differential Equations, vol. 99 (Springer-Verlag, 1993), ISBN 978-1-4612-8741-4, arXiv:1011.1669v3, URL http://link.springer.com/10. 1007/978-1-4612-4342-7. I] IV

${ }^{6}$ T. Erneux, Applied Delay Differential Equations, vol. 3 of Surveys and Tutorials in the Applied Mathematical Sciences (Springer, 2009).

${ }^{7}$ T. Erneux, J. Javaloyes, M. Wolfrum, and S. Yanchuk, Chaos An Interdiscip. J. Nonlinear Sci. 27, 114201 (2017), ISSN 1054-1500, URL http://aip.scitation .org/doi/10.1063/1.5011354

${ }^{8} \mathrm{~S}$. Yanchuk and G. Giacomelli, J. Phys. A Math. Theor. 50, 103001 (2017), ISSN 1751-8113, URL http://stacks.iop.org/1751-8121/50/i=10/a=103001?key= crossref.f760c062e912b820ac69c9174ac61305 [] 
${ }^{9}$ D. Brunner, B. Penkovsky, B. A. Marquez, M. Jacquot, I. Fischer, and L. Larger, J. Appl. Phys. 124, 152004 (2018), ISSN 10897550. I. II A

${ }^{10}$ L. Larger, A. Baylón-Fuentes, R. Martinenghi, V. S. Udaltsov, Y. K. Chembo, and M. Jacquot, Phys. Rev. X 7, 1 (2017), ISSN 21603308. []

${ }^{11}$ A. Röhm and K. Lüdge, J. Phys. Commun. 2, 085007 (2018). I IIA

${ }^{12}$ Y. Chen, L. Yi, J. Ke, Z. Yang, Y. Yang, L. Huang, Q. Zhuge, and W. Hu, Opt. Express 27, 27431 (2019), ISSN 1094-4087. II IIA

${ }^{13}$ C. Sugano, K. Kanno, and A. Uchida, IEEE J. Sel. Top. Quantum Electron. 26, 1 (2020), ISSN 21910359. I]

${ }^{14}$ B. Penkovsky, X. Porte, M. Jacquot, L. Larger, and D. Brunner, Phys. Rev. Lett. 123, 054101 (2019), 1902.05608, URL http://arxiv.org/abs/1902.05608http: //dx.doi.org/10.1103/PhysRevLett.123.054101 [I] II A] II A

${ }^{15}$ C. Gallicchio, A. Micheli, and L. Silvestri, Neurocomputing 298, 34 (2018), ISSN 18728286. I]

${ }^{16} \mathrm{C}$ Gallicchio and A. Micheli, in Int. Work. Artif. Neural Networks (Springer, 2019), pp. 480491.

${ }^{17}$ C. Gallicchio, A. Micheli, and L. Pedrelli, Neurocomputing 268, 87 (2017), ISSN 18728286. II

${ }^{18}$ L. Keuninckx, J. Danckaert, and G. Van der Sande, Cognit. Comput. 9, 315 (2017), ISSN 18669964. II

${ }^{19}$ M. Freiberger, S. Sackesyn, C. Ma, A. Katumba, P. Bienstman, and J. Dambre, IEEE J. Sel. Top. Quantum Electron. 26, 1 (2019). I

${ }^{20}$ A. Argyris, J. Cantero, M. Galletero, E. Pereda, C. R. Mirasso, I. Fischer, and M. C. Soriano, IEEE J. Sel. Top. Quantum Electron. 26, 1 (2020), ISSN 21910359. II A

${ }^{21}$ L. Larger, M. C. Soriano, D. Brunner, L. Appeltant, J. M. Gutierrez, L. Pesquera, C. R. Mirasso, and I. Fischer, Opt. Express 20, 3241 (2012).
${ }^{22}$ M. C. Soriano, S. Ortín, D. Brunner, L. Larger, C. R. Mirasso, I. Fischer, and L. Pesquera, Opt. Express 21, 12 (2013), ISSN 1094-4087.

${ }^{23}$ M. C. Soriano, D. Brunner, M. Escalona-Morán, C. R. Mirasso, and I. Fischer, Front. Comput. Neurosci. 9, 68 (2015), ISSN 1662-5188, URL https://www.frontiersin.org/article/ 10.3389/fncom. 2015.00068

${ }^{24}$ G. Van Der Sande, D. Brunner, and M. C. Soriano, Nanophotonics 6, 561 (2017), ISSN 21928614. II A

${ }^{25}$ X. Gao, Complexity 2019, 1 (2019), ISSN 1076-2787. II A

${ }^{26}$ K. Ikeda, Opt. Commun. 30, 257 (1979), ISSN 00304018. III A

${ }^{27}$ H. Jaeger and H. Haas, Science (80-. ). 304, 78 (2004). II B VIA

${ }^{28}$ J. Snoek, H. Larochelle, and R. P. Adams, Adv. Neural Inf. Process. Syst. 4, 2951 (2012), ISSN 10495258, 1206.2944. II B

${ }^{29}$ J. Dambre, D. Verstraeten, B. Schrauwen, and S. Massar, Sci. Rep. 2, 1 (2012), ISSN 20452322. III, IV VD C C

${ }^{30}$ J. Sieber, K. Engelborghs, T. Luzyanina, G. Samaey, and D. Roose (2014), 1406.7144, URL http://arxiv.org/abs/1406. 7144 III

${ }^{31}$ Note1, the lower input gain $\kappa_{1}$ was chosen to create low-degree $\mathrm{MC}$, because higher degrees are computationally expensive. IV]

${ }^{32}$ W. R. Sweeney, C. W. Hsu, S. Rotter, and A. D. Stone, Phys. Rev. Lett. 122, 93901 (2019), ISSN 10797114, 1807.08805, URL https://doi.org/10.1103/PhysRevLett.122.093901 IV]

${ }^{33}$ S. Ruschel and S. Yanchuk, Philos. Trans. R. Soc. A 377, 20180118 (2019). IV

${ }^{34}$ F. Köster, D. Ehlert, and K. Lüdge, Cognit. Comput. (2020). V VI

${ }^{35}$ K. Harkhoe and G. Van der Sande, Photonics 6, 124 (2019), ISSN 23046732. VVI

${ }^{36}$ F. Stelzer, A. Röhm, K. Lüdge, and S. Yanchuk, Neural Networks 124, 158 (2019), ISSN 18792782, 1905.02534, URL http://arxiv.org/abs/1905.02534\{\%\}0Ahttp://dx.doi. org $/ 10.1016 / j$.neunet.2020.01.010. VI 